PHYSICAL REVIEW D 96, 119901(E) (2017)

\title{
Erratum: Prompt photon yield and elliptic flow from gluon fusion induced by magnetic fields in relativistic heavy-ion collisions [Phys. Rev. D 96, 014023 (2017)]
}

\author{
Alejandro Ayala, Jorge David Castaño-Yepes, C. A. Dominguez, L. A. Hernández, \\ Saúl Hernández-Ortiz, and María Elena Tejeda-Yeomans \\ (Received 13 November 2017; published 11 December 2017)
}

DOI: 10.1103/PhysRevD.96.119901

The computation of the matrix element for the process $g+g \rightarrow \gamma$ published in Ref. [1] contains two errors in Eq. (9) that lead to a change of the final expression for the photon yield and $v_{2}$ induced by this process.

In order to compute the loop integrals in the longitudinal momentum, we introduced a Feynman parametrization. After computing the traces and shifting the integration momentum, we obtained integrals of the form

$$
\begin{aligned}
I & =\int_{0}^{1} d x \int \frac{d^{2} l_{\|}}{(2 \pi)^{2}} \frac{2 l_{\|}^{\mu} l_{\|}^{\nu}-l_{\|}^{2} g_{\|}^{\mu \nu}-x(x-1)\left(p_{\|}^{2} g_{\|}^{\mu \nu}-2 p_{\|}^{\mu} p_{\|}^{\nu}\right)}{\left[l_{p}^{2}-\Delta(x)\right]^{2}}, \\
& =\frac{(-1)}{(4 \pi)^{d / 2}} \frac{i}{\Gamma(2)} \int_{0}^{1} d x\left\{\frac{(1-d / 2) \Gamma(1-d / 2)}{\Delta^{1-d / 2}} g_{\|}^{\mu \nu}+\frac{\Gamma(2-d / 2)}{\Delta^{2-d / 2}} x(x-1)\left(p_{\|}^{2} g_{\|}^{\mu \nu}-2 p_{\|}^{\mu} p_{\|}^{\nu}\right)\right\},
\end{aligned}
$$

with $\Delta(x)=x(x-1) p_{\|}^{2}$.

The first error was to set the dimension $d=2$ in the first term of the integrand in the above expression. The correct treatment is to first combine the terms and at the end set $d=2$. This last procedure leads to the expression

$$
I=-\frac{i}{4 \pi} \int_{0}^{1} d x\left[g_{\|}^{\mu \nu}+\frac{x(x-1) p_{\|}^{2}}{\Delta(x)}\left(g_{\|}^{\mu \nu}-2 \frac{p_{\|}^{\mu} p_{\|}^{\nu}}{p_{\|}^{2}}\right)\right]=-\frac{i}{2 \pi}\left(g_{\|}^{\mu \nu}-\frac{p_{\|}^{\mu} p_{\|}^{\nu}}{p_{\|}^{2}}\right) .
$$

Therefore, Eq. (9) in Ref. [1] should be replaced by

$$
\begin{aligned}
\tilde{M}= & -i(2 \pi)^{4} \delta^{(4)}(q-k-p) \frac{\left|q_{f}\right| g^{2} \delta^{c d} e^{f\left(p_{\perp}, k_{\perp}\right)}}{32 \pi(2 \pi)^{8}}\left\{\left(g_{\|}^{\mu \alpha}-\frac{p_{\|}^{\mu} p_{\|}^{\alpha}}{p_{\|}^{2}}\right) h^{\nu}(a)-\left(g_{\|}^{\mu \nu}-\frac{p_{\|}^{\mu} p_{\|}^{\nu}}{p_{\|}^{2}}\right) h^{\alpha}(a)\right. \\
& \left.+\left(g_{\|}^{\mu \nu}-\frac{k_{\|}^{\mu} k_{\|}^{\nu}}{k_{\|}^{2}}\right) h^{\alpha}(b)-\left(g_{\|}^{\alpha \nu}-\frac{k_{\|}^{\alpha} k_{\|}^{\nu}}{k_{\|}^{2}}\right) h^{\mu}(b)+\left(g_{\|}^{\alpha \nu}-\frac{q_{\|}^{\alpha} q_{\|}^{\nu}}{q_{\|}^{2}}\right) h^{\mu}(c)-\left(g_{\|}^{\mu \alpha}-\frac{q_{\|}^{\mu} q_{\|}^{\alpha}}{q_{\|}^{2}}\right) h^{\nu}(c)\right\} \\
& \times \epsilon_{\mu}\left(\lambda_{p}\right) \epsilon_{\nu}\left(\lambda_{k}\right) \epsilon_{\alpha}\left(\lambda_{q}\right) .
\end{aligned}
$$

Notice that Eq. (3) implies that the matrix element for the process is fully transverse with respect to the components of the particle's momenta along the direction of the magnetic field. This means that the polarization of both the gluons and the photon is oriented in the direction perpendicular to the plane defined by the magnetic field and the particles' direction of motion. Therefore, there is only one polarization to be summed over, and Eq. (11) in Ref. [1] becomes

$$
\sum_{\text {pol }}|\tilde{M}|^{2}=(2 \pi)^{4} \delta^{(4)}(q-k-p) \mathcal{V} \tau_{s} \sum_{\text {pol }}|\mathcal{M}|^{2} .
$$

The second error was made while performing the algebra that leads to the expressions for the vectors $a_{i}, b_{i}, c_{i}$. The correct expressions should be $a_{i}=p_{i}+2 k_{i}+i \epsilon_{i m} p_{m}, b_{i}=2 p_{i}+k_{i}-i \epsilon_{i m} k_{m}$, and $c_{i}=k_{i}-p_{i}+i \epsilon_{i m}\left(p_{m}+k_{m}\right)$.

When correcting these errors, the final result that should appear instead of Eq. (12) in Ref. [1] is

$$
\sum_{\mathrm{pol}}|\mathcal{M}|^{2}=\frac{2 q_{f}^{2} \alpha_{\mathrm{em}} \alpha_{\mathrm{s}}^{2} q_{\perp}^{2}}{\pi \omega_{q}^{2}}\left(2 \omega_{p}^{2}+\omega_{k}^{2}+\omega_{p} \omega_{k}\right) \exp \left\{-\frac{q_{\perp}^{2}}{\left|q_{f} B\right| \omega_{q}^{2}}\left(\omega_{p}^{2}+\omega_{k}^{2}+\omega_{p} \omega_{k}\right)\right\} .
$$



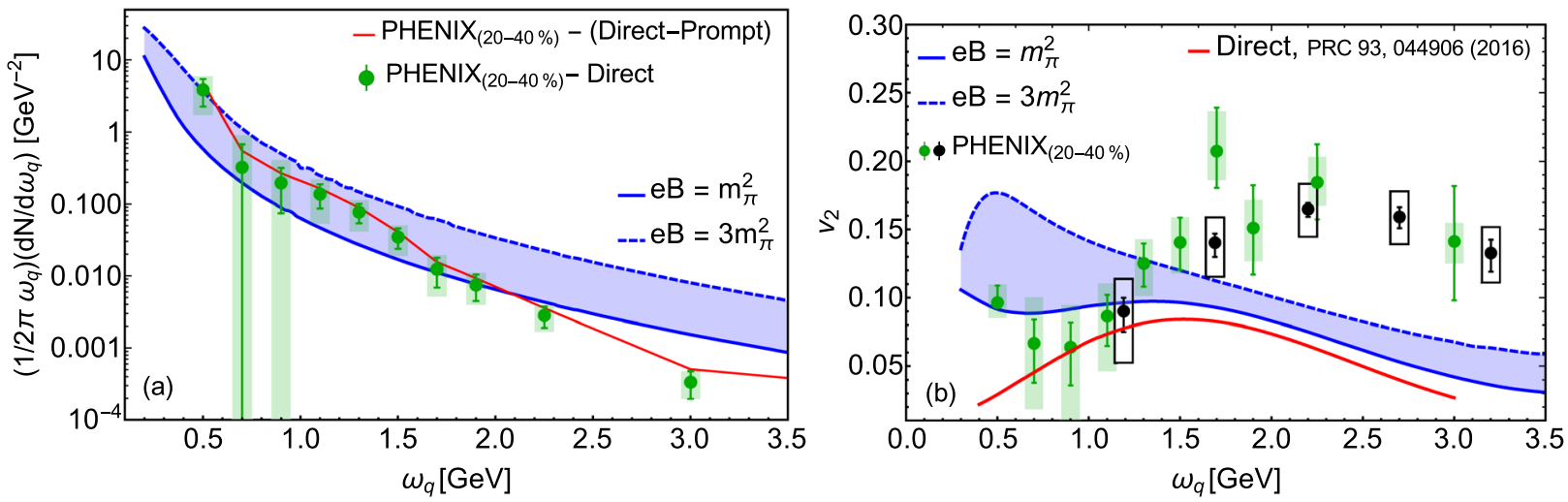

FIG. 2. (a) Difference between PHENIX photon invariant momentum distribution [2] and direct (points) or direct minus prompt (zigzag) photons from Ref. [3] compared to the yield from the present calculation. (b) Harmonic coefficient $v_{2}$ combining the calculation of Ref. [3] and the present calculation compared to PHENIX data [4]. Curves are shown as functions of the photon energy for central rapidity and the centrality range $20 \%-40 \%$. Only the experimental error bars are shown. The bands show variations of the parameter $e B$ within the indicated ranges and computed with $\alpha_{s}=0.3, \Lambda_{s}=2 \mathrm{GeV}, \eta=3, \Delta \tau_{s}=1.5 \mathrm{fm}, R=7 \mathrm{fm}, \beta=0.25$ and $\chi=0.8$.
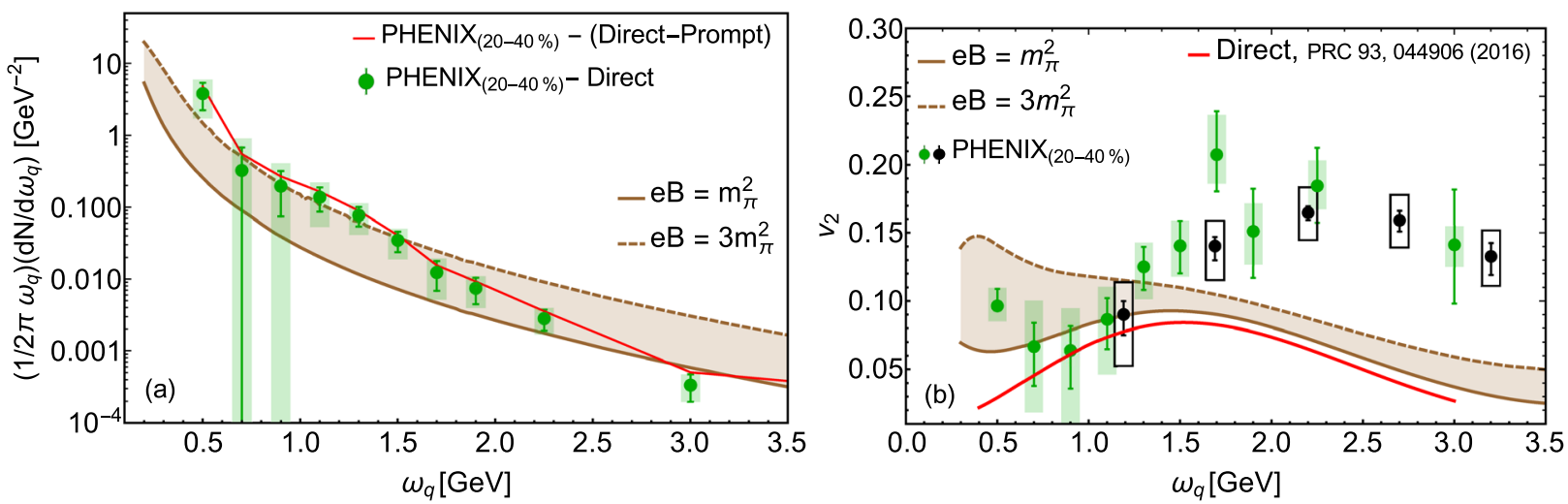

FIG. 3. (a) Difference between PHENIX photon invariant momentum distribution [2] and direct (points) or direct minus prompt (zigzag) photons from Ref. [3] compared to the yield from the present calculation. (b) Harmonic coefficient $v_{2}$ combining the calculation of Ref. [3] and the present calculation compared to PHENIX data [4]. Curves are computed with the same set of parameters as Fig. 1 but with $\beta=0$.

These changes translate into changes for the yield and $v_{2}$ for photon production from gluon fusion. The new plots, which substitute Figs. 2 and 3 in Ref. [1] respectively, are as above.

Notice that the corrections make the description of the excess photon yield and $v_{2}$ to be in better agreement with data, particularly for the low $\omega_{q}$ region of the spectrum.

[1] A. Ayala, J. D. Castaño-Yepes, C. A. Dominguez, L. A. Hernández, S. Hernández-Ortiz, and M. E. Tejeda-Yeomans, Phys. Rev. D 96, 014023 (2017).

[2] A. Adare et al. (PHENIX Collaboration), Phys. Rev. C 91, 064904 (2015).

[3] J.-F. Paquet, C. Shen, G. S. Denicol, M. Luzum, B. Schenke, S. Jeon, and C. Gale, Phys. Rev. C 93, 044906 (2016).

[4] A. Adare et al. (PHENIX Collaboration), Phys. Rev. C 94, 064901 (2016). 